\title{
Indication for management of oropharyngocutaneous fistulas after head and neck reconstruction using a "stick-shaped platysma flap" technique
}

\author{
Mari Matsuura ${ }^{1}$, Hideaki Rikimaru ${ }^{2}$, Yukiko Rikimaru-Nishi ${ }^{3}$, Hisashi Migita ${ }^{3}$, Hiroaki \\ Tanaka $^{3}$, Mai Oyama ${ }^{3}$, and Kensuke Kiyokawa ${ }^{3}$ \\ ${ }^{1}$ Kurume Daigaku - Asahimachi Campus \\ ${ }^{2}$ Kurume University School of Medicine \\ ${ }^{3}$ Kurume University - Asahimachi Campus
}

March 5, 2021

\begin{abstract}
Oropharyngocutaneous fistula is an unfavorable complication that may occur after head and neck reconstruction. The authors used a "stick-shaped platysma flap" technique to close these narrow and deep intractable fistulas. It is a highly efficient and straightforward method to close intractable fistulas with minimal morbidity.
\end{abstract}

\section{INTRODUCTION}

Oropharyngocutaneous fistula after head and neck reconstruction occurs frequently ${ }^{1}$ and is one of the most intractable complications that can negatively affect patient quality of life, due primarily to dysphagia. Factors contributing to its intractability are as follows: (1) comparatively large numbers of bacteria exist in the oral cavity and pharynx, disrupting wound healing by way of either infection or colonization; (2) influx of saliva and swallowing pressure can impede wound healing; (3) orocutaneous fistulas are often located beneath the mandible, where the cortical bone can be an obstacle to wound healing. Cortical bone exposure requires coverage with blood-rich tissue; (4) preoperative radiotherapy and/or chemotherapy may lead to poor blood perfusion and the production of scar tissue, which hamper wound bed preparation. ${ }^{2}$ Also, when postoperative radiotherapy is added to patient treatment, a fistula may become even more intractable and may lead to osteomyelitis of the mandible. Clearly, postoperative fistulas must be closed in short term. ${ }^{3}$

Moreover, Singh et al. reported that the present scenario of the COVID-19 pandemic crisis requires alternative locoregional options for flap reconstruction in the head and neck rather than free flaps due to the increased emphasis on less operative time, shorter flap harvest time, and less postoperative complications and shorter hospital stay. ${ }^{4}$

In our experience, four patients have undergone successful fistula repair with use of our novel stick-shaped platysma flap technique from June 2016 to April 2020 (Table 1). Herein, we report representative cases.

\section{CASES}

2.1 Case 1: A 52-year-old man, after total glossectomy and rectus abdominal muscle (RAM) flap reconstruction for a carcinoma of the tongue (pT3N0M0)

The patient underwent total glossectomy and RAM reconstruction, and subsequently developed a hematoma in the right submandibular region. Nine days postsurgery, incision and drainage was implemented, and at Day 
24 the patient developed a salivary fistula from the right lower jaw into the oral cavity. Since spontaneous closure was difficult to achieve due to swallowing pressure and the presence of dead space, the authors chose to perform the novel stick-shaped platysma flap procedure under local anesthesia at postoperative day 25. Surgical probe revealed a fistula extending from the submandibular region to the right second molar, measuring $10 \mathrm{~mm}$ in width and $35 \mathrm{~mm}$ in length (Fig. 1A). A stick-shaped flap was designed in accordance to the measured dimensions $(10 \mathrm{~mm} \times 45 \mathrm{~mm})$ in the mental region neighboring the fistula (Fig. 1B). The skin incision was made along the design and the flap was dissected including the platysma and underlying loose connective tissue. Particular attention is needed as the loose connective tissue is thought to be the investing layer of cervical fascia, which includes the mandibular branch of the facial nerve. The resulting thickness of the flap, from the epidermis to loose connective tissue, and width of the flap were both approximately $1 \mathrm{~cm}$. Finally, the flap was formed into a stick shape. Next, the flap was transposed and inserted into the fistula. The tip of the flap was sutured to facilitate insertion, and once the flap was pulled into the fistula, the skin margins were marked at the oral and extraoral sites. Then, the middle part of the flap, between the indicated margins, was de-epithelialized (Fig. 2B, C). The inner surface of the fistula was debrided and refreshed with a curette. The flap was again placed into the fistula; hence, the fistula was filled with the middle, de-epithelialized expanse of the flap. As no margin was available for suture at the tip, the flap was placed without suture and the base of the flap was fixed to the skin surrounding the fistula with non-absorbable monofilament sutures 5-0 (Nylon ${ }^{\circledR}$, Bear Medic Corporation, Tokyo, Japan) to prevent the flap from slipping (Fig. 1C). The donor site was closed by direct suture and a split-thickness skin graft (STSG) using the epithelium obtained from the middle area of the flap (Fig. 1C). At 4 days after surgery, the tip of the flap inside the oropharyngeal region became necrotic. Necrotic tissue was debrided as needed, and at postoperative day 15 that region was epithelialized and successfully healed. Furthermore, the flap survived after postoperative radiation therapy (60 Gy). The patient has experienced no recurrence of fistula or ulceration at 12 months after undergoing the stick-shaped platysma flap procedure (Fig. 1D) (Table 1).

\subsection{Case 2: A 77-year-old man, after subtotal glossectomy and RAM flap reconstruction for a} carcinoma of the tongue (pT3N0M0)

The patient underwent radiation therapy (61.2 Gy) after subtotal glossectomy. In addition, hypopharyngeal cancer was detected 10 months after surgery, and a total laryngectomy and partial pharyngectomy were performed. No reconstructive surgery was carried out, and the remaining pharynx was repaired by primary closure. One month following these procedures an orocutaneous fistula developed, which was subsequently closed using a left pectoralis major myocutaneous (PMMC) flap. Nine years and 4 months after subtotal glossectomy, the patient developed a recurrence of tongue cancer, and total glossectomy and reconstruction with a right PMMC flap were performed. Eleven days postoperative an orocutaneous fistula from the left side of the neck through the oral cavity was noted, and the stick-shaped platysma flap procedure was performed at 17 days postoperative. The fistula spanned the left neck region to the left molar and measured $7 \mathrm{~mm}$ and 25 $\mathrm{mm}$ in width and length, respectively (Fig. 3A). A stick-shaped flap measuring $10 \mathrm{~mm} \times 45 \mathrm{~mm}$ was designed in the left mental region neighboring the fistula (Fig. 3B), and the flap was transposed into the fistula. The tip of the flap was exposed to the intraoral side of the fistula and secured with 5-0 Vicryl ${ }^{\circledR}$ (polyglactin/polyL-lactic acid) braided absorbable sutures (Johnson \& Johnson Medical GmbH, Norderstedt, Germany) (Fig. 1C). Another transposed flap below the fistula was transplanted to the fistula aperture. The donor site and exposed surface were closed by STSG using the epithelium removed from the middle part of the stick-shaped flap (Fig. 3C, D). Necrotic tissue on the tip of the flap was debrided as needed on postoperative day 7 , and the wound was completely cured on postoperative day 21 . The patient's postoperative course has been uneventful for 3.5 years (Fig. 3D) (Table 1).

\subsection{Case 3: A 66-year-old man, after extended resection and RAM flap reconstruction for} oropharyngeal cancer (pT4aN1M0)

A 66-year-old man underwent extended resection and RAM flap reconstruction for middle pharyngeal cancer (pT4aN1M0). At postoperative day 9, the patient developed a hematoma and abscess in the right submandibular region, which were drained with a skin incision. The conservative treatment did not achieve improve- 
ment, and a fistula and dead space developed. At postoperative day 21, fistula repair was implemented under general anesthesia. Surgical examination revealed a fistula between the right neck region and the right root of the tongue, measuring $9 \mathrm{~mm}$ wide and $20 \mathrm{~mm}$ long (Fig. 4A). A transposed flap measuring $10 \mathrm{~mm} \times 40 \mathrm{~mm}$ was designed in the right neck region neighboring the fistula (Fig. 4B) and the stick-shaped flap procedure was performed. The mucosa on the inside surface of the fistula was partially split to create adequate space for suturing. The donor site was closed with meshed STSG using femoral skin since de-epithelialized epidermis derived from the flap was not available (Fig. 4C). The patient's postoperative course was uneventful. The flap was well tolerated, and the fistula was successfully repaired. After postoperative radiation therapy (60 Gy) there remains no evidence of recurrence for 3 years and 9 months (Fig. 4D) (Table 1).

\section{DISCUSSION}

Flaps with favorable blood supply are essential for successful closure of intractable fistulas. After head and neck tumor resection, free flaps, such as the radial forearm flap ${ }^{5}$ and anterolateral thigh flap, ${ }^{6,7}$ or regional flaps such as the PMMC flap and supraclavicular flap have been popularly applied for soft tissue reconstruction. ${ }^{8}$ However, most of these flaps are not suitable for narrow fistulas, in neither size nor shape. The novel stick-shaped platysma flap is a suitable surgical treatment to fit in these small and narrow fistulas. Also, this method allows surgeons to preserve more options for another reconstruction in case of recurrence. Further, our method affords additional advantages including shorter operation time, less risk of complication without surgical involvement of tissue around the fistula, easier to carry out without microsurgical technique, and less donor morbidity compared to other free flaps.

With regard to blood supply, this method has the following unique aspects: it includes platysma muscle; it can partially be a composite graft.

Generally, local and direct flaps, which have random-pattern blood perfusion without an axial vascular system, are limited to a safe length-to-width ratio of $1: 1 .{ }^{9}$ In the face and scalp, the richness of the skin circulation, even in the absence of an anatomically recognized axial system, permits length-to-width ratio restrictions to be somewhat relaxed, frequently allowing the flap to be designed as a rectangle rather than as a square. The ratio considered to be safe depends on, to a considerable extent, vascularity of the site from which the flap is obtained, whether the flap is truly random or has an axial element, and whether it is a skin or fasciocutaneous flap. In the novel method presented here, flaps including the platysma muscle are thick enough to be configured into a "stick shape." Describing flaps in a systematic manner is difficult because some belong to more than one category. However, when an investing layer of deep fascia is included, the flap is distinguished as a fasciocutaneous flap rather than a skin flap. ${ }^{9}$ This also accounts for the comparatively good blood perfusion in the stick-shaped flap compared to a mere local skin flap.

Platysma muscle has often been used in head and neck reconstruction, ${ }^{10}$ and it has rich blood perfusion with multiple blood supply. ${ }^{11}$ The platysma myocutaneous flap derives its arterial blood supply predominantly from the submental branch of the facial artery. Additional blood supply comes from the transverse cervical vessels, the thyroid vessels, the occipital vessels, and the posterior auricular vessels. ${ }^{12}$ In the detailed anatomical study of the platysma flap, ${ }^{13}$ it was reported that the platysma was located within the subcutaneous adipofascial tissue, and the vascular system of the platysma was different from that of the musculoskeletal muscles: The platysma has a polygonal arterial plexus instead of a large nutrient artery that runs long. The platysma flap should be considered fasciocutaneous rather than musculocutanous, and it should usually be elevated with the deep adipofascial tissue under the platysma. Likewise, our stick-shaped platysma flap also requires inclusion of the adipofascial tissue under the platysma muscle to obtain favorable blood supply.

Furthermore, the stick-shaped flap has another feature that pertains to blood supply. De-epithelialization of the middle part of the flap serves to facilitate omnidirectional contact to the inner surface of the fistula. Wide contact to intact tissue enables rapid re-perfusion of blood circulation and promotes survival of the intraoral side of the composite graft in case of less robust blood perfusion from the base of the flap to that area. The very tip of the stick-shaped flap often becomes necrotic but undergoes re-epithelialization following debridement making it possible to achieve complete fistula closure (Fig. 5). Correspondingly, in the 
similar technique, the cervical island skin flap, the very tip is reported to become necrotic and epithelializes spontaneously. ${ }^{14}$ Spontaneous healing occurs surprisingly quickly in the intraoral environment, and the result when healing is complete can be unexpectedly good. ${ }^{9}$

The novel stick-shaped platysma flap method is a highly feasible and efficient treatment for intractable fistulas after head and neck reconstruction. It yields minimal morbidity, is comparatively simple to perform, and results in favorable outcomes.

\section{REFERENCES}

1. Sá Breda M, Castro Silva J, Monteiro E. Infections complications in head and neck surgery: Porto Oncology Centre retrospective analysis. Acta Otorrinolaringol Esp. 2019;70:6-15.

2. Watson JS. Experimental microvascular anastomoses in radiated vessels: a study of the patency rate and the histopathology of healing.Plast Reconstr Surg. 1979;63:525-533.

3. Paydarfar JA, Birkmeyer NJ. Complications in head and neck surgery: a meta-analysis of postlaryngectomy pharyngocutaneous fistula.Arch Otolaryngol Head Neck Surg. 2006;132:67-72.

4. Singh A, Chopra A, Chaudhary S, Venkatasubramaniyan M, Agarwal KJM. Modified submental platysmal adipomyofascial flap; is it a reliable alternate reconstructive option for small- to mid- sized defects especially in male patients with oral/oropharungeal cancer? Eur Arch of Otorhinolaryngol. 2020 .

5. Evans GR, Schusterman MA, Kroll SS, et al. The radial forearm free flap for head and neck reconstruction: a review. Am J Surg.1994;168(5):446-450.

6. Liu WW, Li H, Guo ZM, et al. Reconstruction of soft-tissue defects of the head and neck: radial forearm flap or anterolateral thigh flap? Eur Arch Otorhinolaryngol. 2011;268(12):1809-1812.

7. Khadakban D, Kudpaje A, Thankappan K, et al. Reconstructive Indications of Anterolateral Thigh Free Flaps in Head and Neck Reconstruction. Craniomaxillofac Trauma Reconstr.2016;9(1):40-45.

8. Squaquara R, Kim Evans KF, Spilimbergo SP, Mardini S. Intraoral reconstruction using local and regional flaps. Semin Plast Surg. 2010;24(2):198-211.

9. McGregor IA, McGregor AD. Fundamental Techniques of Plastic Surgery and Their Surgical Applications 10th Edition. Edinburgh: Churchill Livingstone; 1989. p 61-120.

10. Farr HW, Jean-Gillies B, Die A. Cervical island skin flap repair of oral and pharyngeal defects in the composite operation for cancer.Am J Surg. 1969;118:759.

11. Ruark DS, McClairen WC, Schlehaider UK, Abdel-Misih RZ. Head and neck reconstruction using the platysma myocutaneous flap. Am J Surg.1993;165:713-718.

12. McGuirt WF, Matthews BL, Brody JA, May JS. Platysma myocutaneous flap: caveats reexamined. Laryngoscope. 1991;101(11):1238-1244.

13. Imanishi N, Nakajima H, Kishi K, et al. Is the platysma flap musculous? Angiographic study of the platysma. Plast Reconstr Surg. 2005;115(4):1018-1024.

14. Kudo K, Tsuge N, Miyasawa M, et al. Immediate reconstruction following resection of oral cancers using cervical island skin flap. Jpn J Oral Maxillofac Surg. 1985;31:661-666.

\section{FIGURE LEGENDS}

Figure 1. Preparation of a flap. (A) Flap design. (B) Diagram showing the cross-sectional view of flap design. Dissection layer below the superficial cervical fascia (red arrow); (C) Diagram illustrating the flap after skin incision and flap elevation. Path of skin flap insertion (black dotted arrow); (D) Diagram illustrating the flap pulled into a fistula. (1) The base of flap; (2) De-epithelialized area (red dotted arrow); (3) Fistula; (4) Epidermis; (5) Dermis; (6) Subcutaneous tissue; (7) Platysma; (8) Investing layer of cervical fascia, attached to the flap; (9) Muscle layer under the superficial cervical fascia; (10) Oropharyngeal mucosa; (11) Fixed suture. If there is no margin for suture at the intraoral site, the base of the flap is fixed to the surrounding skin of fistula; (12) Secured with suture at the intraoral site; (13) Donor site was closed with primary closure or skin graft.

Figure 2. Case 1: A 52-year-old man with a right submandibular orocuta- 
neous fistula. (A) Preoperative findings. Fistula measured with a surgical probe $\left.{ }^{(}\right)$; (B)Designofastick-shapedflapinthementalregion; (C)Postoperative findings.ST SGobtainedfromdeepithelializedskin $(*) ;$ Thebaseoftheflap(Arrowhead).(D)Findings6monthspost

surgery.Nofistularecurrence.

Figure 3. Case 2: A 77-year-old man with a left mandibular orocutaneous fistula. (A) Stick-shaped flap designed in mental region. Fistula and dead space of mandibular area ()$;(B)$ Elevatedde-epithelializedstickshapedflap (Arrowhead)andtransposedlocal flapbelowthe fistula $(*)$; Intraoperativefindingsofthe $(C)$ frontand $(D)$ leftside. shapedflap (Arrowhead), transposedlocal flap $(*)$.Thedonorsiteandrawsurfaceneighboringthe fistulawererepairedbySTSG surgery.Nofistularecurrence.

Figure 4. Case 3: A 66-year-old man with a right pharyngocutaneous fistula. (A) Fistula and dead space in submandibular region $\left(^{(} ;(B)\right.$ Designofastick-shapedflap; $(C)$ Postoperativefindings.Transplantedstickshapedflap(Arrowhead), meshedSTSG(*), anddrainagetube $\left({ }^{\prime} ;(D)\right.$ Findings6monthspost surgery.Nofistularecurrence.

Figure 5. Blood flow distribution and survival mechanism of stick-shaped flap; (1) Survival area as a fasciocutaneous flap; (2) Possibly survival area as a composite graft in case of unstable blood circulation; (3) Debridement area if necrosis occurs ('.

A

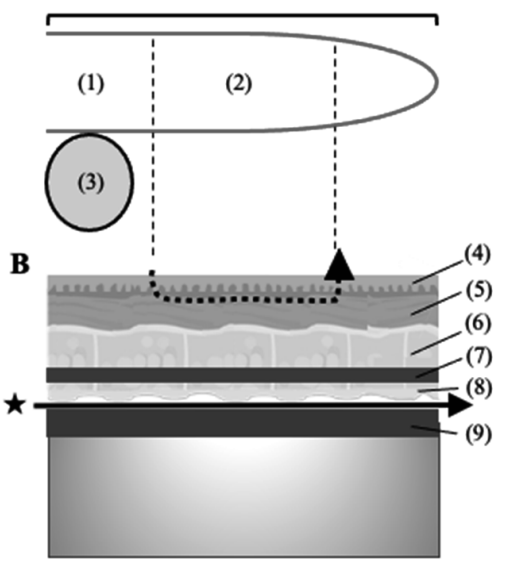

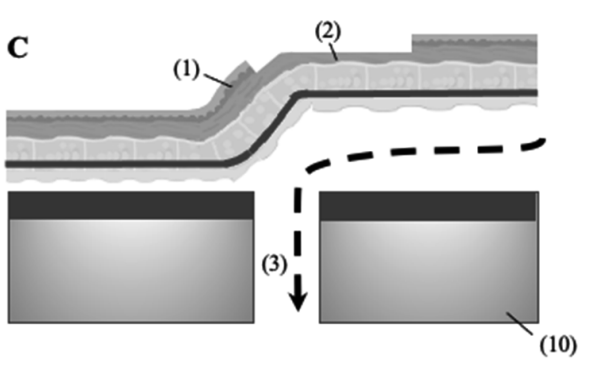

D

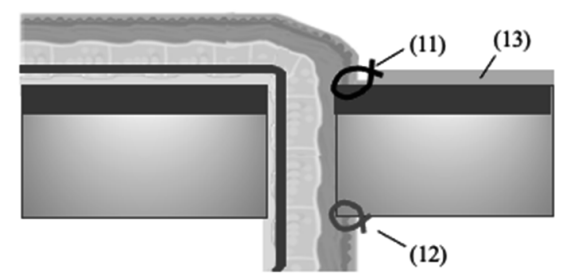



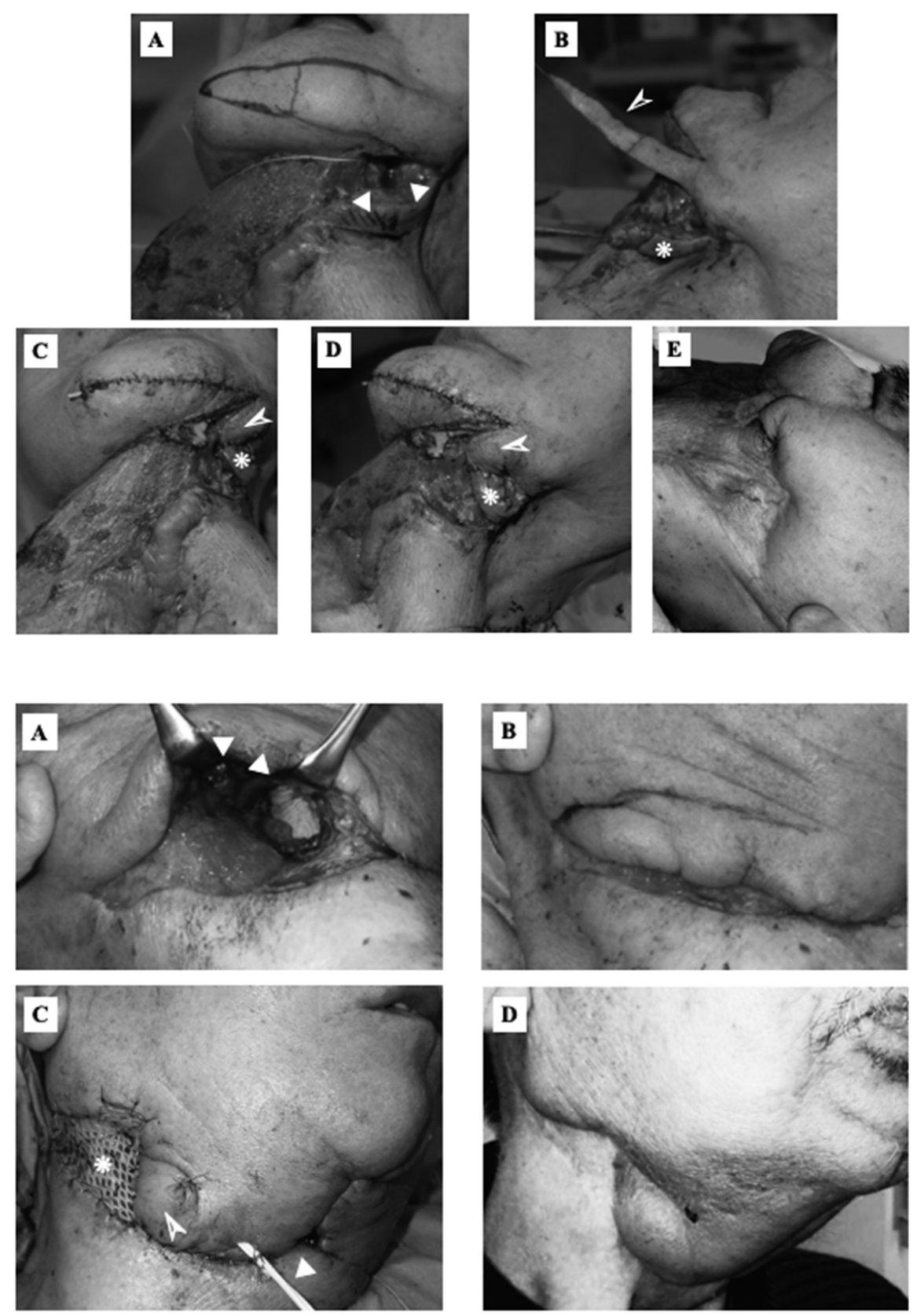


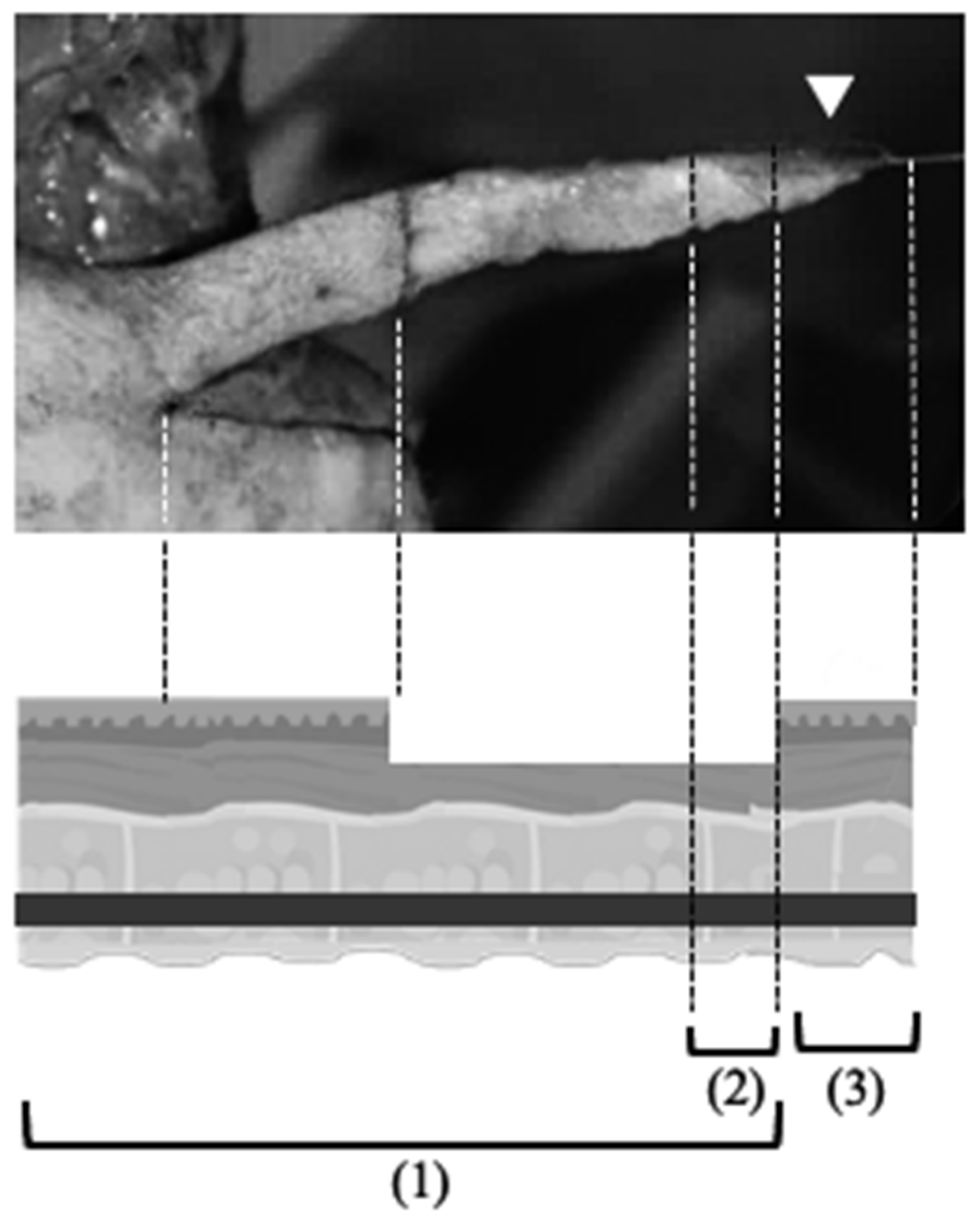

\section{Hosted file}

Table 1.pdf available at https://authorea.com/users/399759/articles/512190-indication-formanagement-of-oropharyngocutaneous-fistulas-after-head-and-neck-reconstruction-using-astick-shaped-platysma-flap-technique 